\title{
APPENDIX II. CARBON AND CARBONATE ANALYSES, LEG 17
}

Gerald W. Bode, Scripps Institution of Oceanography, La Jolla, California

Leg 17 sediments were analyzed for total carbon and acid-insoluble (organic) carbon using a LECO 70 Second Analyzer. The 3-cc sediment samples were first dried at $105^{\circ}$ to $110^{\circ} \mathrm{C}$ and then ground to a homogeneous powder. The ground sediment was redried and two samples, a $0.1-\mathrm{g}$ and a 0.5 -g sample, were than weighed into LECO clay crucibles. The $0.5 \mathrm{~g}$ sample was acidified with dilute hydrochloric acid and washed with distilled water. The sample was then dried and analyzed for acid-insoluble carbon which is labeled in the table as "organic" carbon. The 0.1-g sample was analyzed for total carbon without further treatment. If the result showed less than $10 \%$ $\mathrm{CaCO}_{3}$, an additional 0.5 -g sample was analyzed for greater accuracy. The calcium carbonate percentages were calculated as follows: (\% total C-\% organic C) $\times 8.33=\%$ $\mathrm{CaCO}_{3}$. Although other carbonates may be present, all acid-soluble carbon was calculated as calcium carbonate. All results are given in weight percent (Table 1). Precisions for the analyses are as follows:

Total Carbon:

(1.2 to $12 \%$ ): $\pm 0.2 \%$ (absolute variation)

(0 to $1.2 \%$ ): $\pm 0.04 \%$ (absolute variation)

Organic Carbon: $\pm 0.04 \%$ (absolute variation)

Calcium Carbonate:

(10 to $100 \%$ ): $\pm 2 \%$ (absolute variation)

(0 to $10 \%$ ): $\pm 0.6 \%$ (absolute variation)

Detailed descriptions of the technique and theory may be found in Bader, Gerard, et al. (1970) and Boyce and Bode (1972).

\section{REFERENCES}

Bader, R. G., Gerard, R. D., et al., 1970. Initial Reports of the Deep Sea Drilling Project, Volume IV. Washington (U. S. Government Printing Office), 745 p.

Boyce, R. E. and Bode, G. W., 1972. Carbon and carbonate analyses, Leg 9, Deep Sea Drilling Project: Initial Reports of the Deep Sea Drilling Project, Volume IX. Washington (U.S. Government Printing Office). p. 797.

TABLE 1

Carbon-Carbonate Analyses, Leg 17

\begin{tabular}{ccccc}
\hline $\begin{array}{c}\text { Core, Section, } \\
\text { Top of } \\
\begin{array}{c}\text { Interval } \\
(\mathrm{cm})\end{array}\end{array}$ & $\begin{array}{c}\text { Hole } \\
\text { Depth } \\
(\mathrm{m})\end{array}$ & $\begin{array}{c}\text { Total } \\
\text { Carbon } \\
(\%)\end{array}$ & $\begin{array}{c}\text { Organic } \\
\text { Carbon } \\
(\%)\end{array}$ & $\begin{array}{c}\mathrm{CaCO}_{3} \\
(\%)\end{array}$ \\
\hline Site $\mathbf{1 6 4}$ & & & & \\
& & & & \\
$1-5,50$ & 34.5 & 0.1 & 0.1 & 0 \\
$2-2,50$ & 50.00 & 0.2 & 0.1 & 1 \\
$3-1,50$ & 51.50 & 0.1 & 0.1 & 0 \\
$3-5,116$ & 58.16 & 0.1 & 0.1 & 0 \\
$4-1,91$ & 57.91 & 0.0 & 0.0 & 0 \\
\hline
\end{tabular}

TABLE 1 - Continued

\begin{tabular}{ccccc}
\hline $\begin{array}{c}\text { Core, Section, } \\
\text { Top of } \\
\text { Interval } \\
(\mathrm{cm})\end{array}$ & $\begin{array}{c}\text { Hole } \\
\text { Depth } \\
(\mathrm{m})\end{array}$ & $\begin{array}{c}\text { Total } \\
\text { Carbon } \\
(\%)\end{array}$ & $\begin{array}{c}\text { Organic } \\
\text { Carbon } \\
(\%)\end{array}$ & $\begin{array}{c}\mathrm{CaCO}_{3} \\
(\%)\end{array}$ \\
\hline $6-1,50$ & 75.50 & 0.1 & 0.1 & 1 \\
$7-1,60$ & 84.60 & 0.1 & 0.1 & 0 \\
$7-4,50$ & 89.00 & 0.1 & 0.1 & 0 \\
$7-5,37$ & 90.37 & 0.1 & 0.1 & 0 \\
$8-1,55$ & 93.55 & 0.1 & 0.1 & 0 \\
$8-2,50$ & 95.00 & 0.2 & 0.1 & 1 \\
$8-3,50$ & 96.50 & 0.2 & 0.1 & 1 \\
$8-4,60$ & 98.10 & 0.2 & 0.1 & 1 \\
$10-2,50$ & 114.00 & 0.2 & 0.1 & 1 \\
$10-3,50$ & 115.50 & 0.2 & 0.1 & 0 \\
$10-4,50$ & 117.00 & 0.2 & 0.1 & 1 \\
$11-1,118$ & 123.18 & 0.3 & 0.1 & 1 \\
$13-1,83$ & 137.83 & 0.2 & 0.1 & 1 \\
$25-1,125$ & 253.25 & 0.2 & 0.1 & 1
\end{tabular}

Site 165

$\begin{array}{lrrrr}1-1,50 & 0.50 & 0.3 & 0.1 & 1 \\ 1-1,53 & 0.53 & 4.5 & 0.1 & 36 \\ 1-1,56 & 0.56 & 6.6 & 0.1 & 54 \\ 1-1,59 & 0.59 & 8.2 & 0.1 & 68 \\ 1-1,62 & 0.62 & 9.3 & 0.1 & 77 \\ 1-1,64 & 0.64 & 0.2 & 0.2 & 1 \\ 1-1,106 & 1.06 & 10.6 & 0.1 & 88 \\ 1-2,143 & 2.93 & 10.5 & 0.1 & 87 \\ 1-3,25 & 3.25 & 10.2 & 0.1 & 84 \\ 2-2,50 & 7.00 & 0.2 & 0.1 & 1\end{array}$

Site 165A

\begin{tabular}{lrrll}
$1-2,7$ & 6.57 & 10.7 & 0.1 & 89 \\
$1-2,36$ & 6.86 & 9.7 & 0.1 & 80 \\
$1-2,43$ & 6.93 & 8.6 & 0.1 & 71 \\
$1-5,116$ & 12.16 & 10.5 & 0.1 & 87 \\
$2-1,66$ & 14.66 & 8.9 & 0.1 & 73 \\
$2-3,12$ & 17.12 & 4.5 & 0.1 & 37 \\
$2-3,58$ & 17.58 & 9.1 & 0.1 & 75 \\
$2-5,87$ & 20.87 & 9.3 & 0.0 & 77 \\
$2-5,98$ & 20.98 & 7.8 & 0.0 & 64 \\
$3-3,52$ & 27.52 & 10.3 & 0.0 & 86 \\
$3-3,87$ & 27.87 & 9.7 & 0.0 & 80 \\
$3-4,30$ & 28.80 & 10.5 & 0.0 & 87 \\
$3-4,54$ & 29.04 & 8.1 & 0.0 & 67 \\
$3-5,50$ & 30.50 & 9.7 & 0.0 & 80 \\
$3-5,69$ & 30.69 & 10.2 & 0.0 & 85 \\
$5-2,138$ & 82.88 & 10.1 & 0.0 & 84 \\
$5-3,31$ & 83.31 & 9.1 & 0.0 & 76 \\
$5-4,139$ & 85.89 & 8.6 & 0.0 & 71 \\
$6-1,50$ & 127.50 & 9.4 & 0.0 & 78 \\
$6-2,50$ & 129.00 & 10.0 & 0.0 & 83 \\
$6-3,50$ & 130.50 & 9.9 & 0.0 & 82 \\
$6-4,50$ & 132.00 & 9.5 & 0.0 & 78 \\
\hline
\end{tabular}


TABLE 1 - Continued

\begin{tabular}{|c|c|c|c|c|}
\hline $\begin{array}{l}\text { Core, Section, } \\
\text { Top of } \\
\text { Interval } \\
(\mathrm{cm})\end{array}$ & $\begin{array}{l}\text { Hole } \\
\text { Depth } \\
(\mathrm{m})\end{array}$ & $\begin{array}{l}\text { Total } \\
\text { Carbon } \\
(\%)\end{array}$ & $\begin{array}{c}\text { Organic } \\
\text { Carbon } \\
(\%)\end{array}$ & $\begin{array}{c}\mathrm{CaCO}_{3} \\
(\%)\end{array}$ \\
\hline $\begin{array}{l}6-5,50 \\
6-6,80\end{array}$ & $\begin{array}{l}133.50 \\
135.30\end{array}$ & $\begin{array}{l}9.7 \\
8.5\end{array}$ & $\begin{array}{l}0.0 \\
0.0\end{array}$ & $\begin{array}{l}80 \\
71\end{array}$ \\
\hline $\begin{array}{l}7-1,50 \\
7-2,50 \\
7-6,140\end{array}$ & $\begin{array}{l}136.50 \\
138.00 \\
144.90\end{array}$ & $\begin{array}{r}10.0 \\
10.5 \\
5.8\end{array}$ & $\begin{array}{l}0.0 \\
0.0 \\
0.0\end{array}$ & $\begin{array}{l}83 \\
87 \\
48\end{array}$ \\
\hline $\begin{array}{l}8-1,10 \\
8-1,18 \\
8-1,38 \\
8-1,61 \\
8-1,80 \\
8-1,101 \\
8-3,16\end{array}$ & $\begin{array}{l}145.10 \\
145.18 \\
145.38 \\
145.61 \\
145.80 \\
146.01 \\
148.16\end{array}$ & $\begin{array}{l}0.1 \\
4.8 \\
9.9 \\
9.8 \\
8.5 \\
6.8 \\
3.7\end{array}$ & $\begin{array}{l}0.0 \\
0.0 \\
0.0 \\
0.0 \\
0.0 \\
0.0 \\
0.0\end{array}$ & $\begin{array}{r}1 \\
40 \\
83 \\
81 \\
71 \\
56 \\
31\end{array}$ \\
\hline $\begin{array}{l}9-1,50 \\
9-1,100 \\
9-2,34 \\
9-2,110 \\
9-3,30 \\
9-3,85 \\
9-3,130 \\
9-4,40 \\
9-4,107 \\
9-5,34 \\
9-5,89 \\
9-6,101 \\
9-6,139 \\
9-6,142.5\end{array}$ & $\begin{array}{l}154.50 \\
155.00 \\
155.84 \\
156.60 \\
157.30 \\
157.85 \\
158.30 \\
158.90 \\
159.57 \\
160.34 \\
160.89 \\
162.51 \\
162.89 \\
162.93\end{array}$ & $\begin{array}{l}3.3 \\
5.5 \\
8.4 \\
5.9 \\
5.0 \\
1.7 \\
1.0 \\
1.8 \\
4.5 \\
4.4 \\
2.7 \\
3.1 \\
6.0 \\
4.5\end{array}$ & $\begin{array}{l}0.0 \\
0.0 \\
0.0 \\
0.0 \\
0.0 \\
0.0 \\
0.0 \\
0.0 \\
0.0 \\
0.0 \\
0.0 \\
0.0 \\
0.0 \\
0.0\end{array}$ & $\begin{array}{r}27 \\
46 \\
70 \\
50 \\
41 \\
14 \\
8 \\
15 \\
38 \\
37 \\
22 \\
26 \\
50 \\
37\end{array}$ \\
\hline $\begin{array}{l}10-1,74 \\
10-2,130 \\
10-2,40 \\
10-2,130 \\
10-3,40 \\
10-3,125 \\
10-4,40 \\
10-4,110 \\
10-5,40 \\
10-5,110 \\
10-6,40 \\
10-6,120\end{array}$ & $\begin{array}{l}201.74 \\
202.30 \\
202.90 \\
203.80 \\
204.40 \\
205.25 \\
205.90 \\
206.60 \\
207.40 \\
208.10 \\
208.90 \\
209.70\end{array}$ & $\begin{array}{l}8.7 \\
7.6 \\
7.2 \\
7.4 \\
6.4 \\
6.8 \\
6.7 \\
6.9 \\
7.0 \\
6.8 \\
7.2 \\
6.9\end{array}$ & $\begin{array}{l}0.0 \\
0.0 \\
0.0 \\
0.0 \\
0.0 \\
0.0 \\
0.0 \\
0.0 \\
0.0 \\
0.0 \\
0.0 \\
0.0\end{array}$ & $\begin{array}{l}72 \\
63 \\
60 \\
62 \\
53 \\
57 \\
55 \\
57 \\
58 \\
57 \\
60 \\
58\end{array}$ \\
\hline $\begin{array}{l}11-1,64 \\
11-1,140 \\
11-2,50 \\
11-2,110 \\
11-3,40 \\
11-3,120 \\
11-4,30 \\
11-4,100 \\
11-5,40 \\
11-5,120\end{array}$ & $\begin{array}{l}210.64 \\
211.40 \\
212.00 \\
212.60 \\
213.40 \\
214.20 \\
214.80 \\
215.50 \\
216.40 \\
217.20\end{array}$ & $\begin{array}{l}5.5 \\
5.6 \\
1.6 \\
4.5 \\
6.3 \\
8.7 \\
7.2 \\
8.7 \\
8.8 \\
8.9\end{array}$ & $\begin{array}{l}0.0 \\
0.0 \\
0.0 \\
0.0 \\
0.0 \\
0.0 \\
0.0 \\
0.0 \\
0.0 \\
0.0\end{array}$ & $\begin{array}{l}45 \\
47 \\
13 \\
38 \\
52 \\
72 \\
60 \\
72 \\
73 \\
74\end{array}$ \\
\hline $\begin{array}{l}12-1,33 \\
12-1,110 \\
12-2,68 \\
12-2,99 \\
12-3,54 \\
12-3,110 \\
12-4,42 . \\
12-4,60 \\
12-4,82 \\
12-5,17 \\
12-5,42 \\
12-5,103\end{array}$ & $\begin{array}{l}219.33 \\
220.10 \\
221.18 \\
221.49 \\
222.54 \\
223.10 \\
223.92 \\
224.10 \\
224.32 \\
225.17 \\
225.42 \\
226.03\end{array}$ & $\begin{array}{l}7.9 \\
6.3 \\
3.8 \\
0.9 \\
0.1 \\
7.7 \\
0.4 \\
0.4 \\
1.7 \\
6.2 \\
1.7 \\
2.5\end{array}$ & $\begin{array}{l}0.0 \\
0.0 \\
0.0 \\
0.0 \\
0.0 \\
0.0 \\
0.0 \\
0.0 \\
0.0 \\
0.0 \\
0.0 \\
0.0\end{array}$ & $\begin{array}{r}66 \\
53 \\
32 \\
8 \\
0 \\
64 \\
3 \\
4 \\
14 \\
52 \\
14 \\
21\end{array}$ \\
\hline $\begin{array}{l}13-1,86 \\
13-1,98 \\
13-1,126\end{array}$ & $\begin{array}{l}228.86 \\
228.98 \\
229.26\end{array}$ & $\begin{array}{l}7.5 \\
0.0 \\
2.9\end{array}$ & $\begin{array}{l}0.0 \\
0.0 \\
0.0\end{array}$ & $\begin{array}{r}62 \\
0 \\
24\end{array}$ \\
\hline
\end{tabular}

TABLE 1 - Continued

\begin{tabular}{lcccr}
\hline $\begin{array}{c}\text { Core, Section } \\
\text { Top of } \\
\text { Interval } \\
(\mathrm{cm})\end{array}$ & $\begin{array}{c}\text { Depth } \\
\text { in Hole } \\
(\mathrm{m})\end{array}$ & $\begin{array}{c}\text { Carbon } \\
\text { Total } \\
(\%)\end{array}$ & $\begin{array}{c}\text { Organic } \\
\text { Carbon } \\
(\%)\end{array}$ & $\begin{array}{c}\mathrm{CaCO}_{3} \\
(\%)\end{array}$ \\
\hline $13-2,24$ & 229.74 & 7.4 & 0.0 & 61 \\
$13-2,96$ & 230.46 & 0.0 & 0.0 & 0 \\
$13-5,50$ & 234.50 & 0.0 & 0.0 & 0 \\
$18-1,82$ & 290.82 & 2.6 & 0.0 & 22 \\
$18-2,103$ & 292.53 & 0.3 & 0.1 & 2 \\
$18-3,50$ & 293.50 & 1.5 & 0.0 & 12 \\
$19-1,86$ & 321.86 & 0.2 & 0.0 & 1 \\
$19-1,94$ & 321.94 & 8.1 & 0.0 & 68 \\
$19-2,113$ & 323.63 & 1.6 & 0.0 & 13 \\
$20-3,140$ & 344.40 & 6.8 & 0.0 & 57 \\
$21-2,51$ & 370.01 & 7.3 & 0.0 & 60 \\
$21-3,33$ & 371.33 & 7.5 & 0.0 & 62 \\
$22-3,6$ & 399.06 & 1.5 & 0.1 & 12
\end{tabular}

Site 166

\begin{tabular}{|c|c|c|c|c|}
\hline $2-1,50$ & 1.50 & 0.1 & 0.1 & 0 \\
\hline $2-2,50$ & 3.00 & 0.3 & 0.1 & 2 \\
\hline $2-3,50$ & 4.50 & 0.1 & 0.1 & 1 \\
\hline $2-4,50$ & 6.00 & 0.2 & 0.0 & 1 \\
\hline $2-5,12$ & 7.12 & 0.2 & 0.1 & 1 \\
\hline $2-6,50$ & 9.00 & 0.2 & 0.1 & 1 \\
\hline $3-3,50$ & 14.50 & 0.2 & 0.1 & 1 \\
\hline $4-1,50$ & 20.50 & 0.5 & 0.0 & 4 \\
\hline $4-2,50$ & 22.00 & 0.4 & 0.0 & 3 \\
\hline $4-3,50$ & 23.50 & 0.7 & 0.0 & 6 \\
\hline $4-4,50$ & 25.00 & 0.4 & 0.1 & 3 \\
\hline $4-5,50$ & 26.50 & 1.8 & 0.0 & 15 \\
\hline $4-6,50$ & 28.00 & 2.6 & 0.0 & 21 \\
\hline $5-1,80$ & 29.80 & 2.4 & 0.0 & 20 \\
\hline $5-2,60$ & 31.10 & 3.1 & 0.0 & 26 \\
\hline $5-3,50$ & 32.50 & 3.2 & 0.0 & 27 \\
\hline $5-4,50$ & 34.00 & 0.9 & 0.1 & 7 \\
\hline $5-5,50$ & 35.50 & 2.1 & 0.1 & 17 \\
\hline $7-1,50$ & 65.50 & 0.8 & 0.1 & 6 \\
\hline $7-2,50$ & 67.00 & 0.7 & 0.1 & 5 \\
\hline $7-3,50$ & 68.50 & 0.1 & 0.1 & 0 \\
\hline $7-4,50$ & 70.00 & 1.9 & 0.1 & 15 \\
\hline $7-5,50$ & 71.50 & 2.8 & 0.0 & 23 \\
\hline $7-6,50$ & 73.00 & 1.4 & 0.1 & 11 \\
\hline $8-1,50$ & 84.50 & 0.6 & 0.1 & 5 \\
\hline $8-2,20$ & 85.70 & 0.6 & 0.1 & 4 \\
\hline $8-3,50$ & 87.50 & 0.1 & 0.1 & 0 \\
\hline $8-4,50$ & 89.00 & 0.1 & 0.0 & 0 \\
\hline $8-5,59$ & 90.59 & 0.0 & 0.0 & 0 \\
\hline $9-1,107$ & 104.07 & 0.1 & 0.1 & 0 \\
\hline $9-2,50$ & 105.00 & 0.1 & 0.1 & 0 \\
\hline $9-3,45$ & 106.45 & 0.1 & 0.1 & 0 \\
\hline $9-4,48$ & 107.98 & 0.0 & 0.0 & 0 \\
\hline $9-5,50$ & 109.50 & 0.1 & 0.0 & 0 \\
\hline $9-6,17$ & 110.67 & 0.9 & 0.1 & 7 \\
\hline $10-2,50$ & 123.00 & 0.0 & 0.0 & 0 \\
\hline $10-3,50$ & 124.50 & 0.6 & 0.1 & 5 \\
\hline $10-4,50$ & 126.00 & 0.4 & 0.1 & 3 \\
\hline $10-5,50$ & 127.50 & 2.5 & 0.1 & 20 \\
\hline $12-1,31$ & 159.31 & 0.0 & 0.0 & 0 \\
\hline $12-1,134$ & 160.34 & 0.0 & 0.0 & 0 \\
\hline $12-2,50$ & 161.00 & 0.0 & 0.0 & 0 \\
\hline $12-3,50$ & 162.50 & 0.3 & 0.1 & 2 \\
\hline $12-4,50$ & 164.00 & 0.0 & 0.0 & 0 \\
\hline
\end{tabular}


TABLE 1 - Continued

\begin{tabular}{ccccc}
\hline $\begin{array}{c}\text { Core, Section, } \\
\text { Top of } \\
\text { Interval } \\
(\mathrm{cm})\end{array}$ & $\begin{array}{c}\text { Hole } \\
\text { Depth } \\
(\mathrm{m})\end{array}$ & $\begin{array}{c}\text { Total } \\
\text { Carbon } \\
(\%)\end{array}$ & $\begin{array}{c}\text { Organic } \\
\text { Carbon } \\
(\%)\end{array}$ & $\begin{array}{c}\mathrm{CaCO}_{3} \\
(\%)\end{array}$ \\
\hline $12-5,50$ & 165.50 & 0.0 & 0.0 & 0 \\
$12-6,50$ & 167.00 & 0.0 & 0.0 & 0 \\
$13-1,50$ & 169.50 & 0.1 & 0.0 & 0 \\
$13-2,50$ & 171.00 & 0.1 & 0.0 & 0 \\
$13-3,50$ & 172.50 & 0.0 & 0.0 & 0 \\
$13-4,50$ & 174.00 & 0.0 & 0.0 & 0 \\
$13-5,72$ & 175.72 & 0.0 & 0.0 & 0 \\
$13-6,35$ & 176.85 & 0.0 & 0.0 & 0 \\
$16-2,50$ & 191.00 & 0.1 & 0.0 & 1 \\
$16-3,50$ & 192.50 & 0.1 & 0.0 & 0 \\
$16-4,50$ & 194.00 & 0.0 & 0.0 & 0 \\
$23-2,32$ & 251.82 & 0.0 & 0.0 & 0 \\
$23-2,89$ & 252.39 & 0.0 & 0.0 & 0 \\
$23-2,134$ & 252.84 & 0.0 & 0.0 & 0 \\
$23-2,137$ & 252.87 & 0.0 & 0.0 & 0 \\
$24-3,102$ & 264.02 & 0.6 & 0.0 & 5 \\
$24-5,109$ & 267.09 & 2.2 & 0.0 & 18 \\
$25-1,88$ & 270.88 & 0.0 & 0.0 & 0 \\
$25-1,122$ & 271.22 & 0.0 & 0.0 & 0 \\
$27-2,38$ & 290.88 & 4.8 & 0.0 & 40 \\
& & & &
\end{tabular}

\section{Site 166A}

$\begin{array}{lrrrr}1-1,2 & 0.02 & 7.8 & 0.1 & 64 \\ 1-1,60 & 0.60 & 0.1 & 0.0 & 1 \\ 1-2,50 & 2.00 & 0.1 & 0.0 & 1 \\ 1-3,75 & 3.75 & 0.1 & 0.0 & 0 \\ 1-3,108 & 4.08 & 11.3 & 0.0 & 94 \\ 1-4,50 & 5.00 & 0.1 & 0.0 & 1\end{array}$

Site 167

$\begin{array}{lrrrr}2-2,50 & 11.00 & 11.3 & 0.0 & 94 \\ 2-5,61 & 15.61 & 11.0 & 0.0 & 92 \\ 2-5,101 & 16.01 & 11.4 & 0.0 & 95 \\ 3-1,50 & 18.50 & 11.3 & 0.0 & 94 \\ 4-6,50 & 74.00 & 11.2 & 0.0 & 93 \\ 7-2,50 & 151.00 & 11.2 & 0.0 & 93 \\ 7-5,100 & 156.00 & 11.3 & 0.0 & 94 \\ 7-5,143 & 156.43 & 10.9 & 0.0 & 91 \\ 8-1,133 & 187.33 & 11.4 & 0.0 & 94 \\ 9-1,50 & 223.50 & 11.2 & 0.0 & 93 \\ 9-2,50 & 225.00 & 11.4 & 0.0 & 95 \\ 9-4,74 & 228.24 & 11.4 & 0.0 & 95 \\ 9-6,50 & 231.00 & 11.6 & 0.0 & 96 \\ 10-2,120 & 262.70 & 11.3 & 0.0 & 94 \\ 10-4,55 & 265.05 & 11.2 & 0.0 & 94 \\ 10-6,50 & 268.00 & 11.4 & 0.0 & 94 \\ 11-1,49 & 297.49 & 11.2 & 0.0 & 93 \\ 11-4,50 & 302.00 & 11.1 & 0.0 & 92 \\ 11-5,50 & 303.50 & 11.1 & 0.0 & 92 \\ 12-2,51 & 336.01 & 11.4 & 0.0 & 95 \\ 12-6,50 & 342.00 & 11.4 & 0.0 & 95 \\ 13-3,41 & 373.41 & 11.3 & 0.0 & 94 \\ 14-4,37 & 411.87 & 11.1 & 0.0 & 92 \\ 18-2,60 & 474.10 & 11.4 & 0.0 & 94 \\ 19-2,50 & 483.00 & 11.2 & 0.0 & 93\end{array}$

TABLE 1 - Continued

\begin{tabular}{|c|c|c|c|c|}
\hline $\begin{array}{l}\text { Core, Section, } \\
\text { Top of } \\
\text { Interval } \\
\text { (cm) }\end{array}$ & $\begin{array}{l}\text { Hole } \\
\text { Depth } \\
\text { (m) }\end{array}$ & $\begin{array}{l}\text { Total } \\
\text { Carbon } \\
(\%)\end{array}$ & $\begin{array}{c}\text { Organic } \\
\text { Carbon } \\
(\%)\end{array}$ & $\begin{array}{c}\mathrm{CaCO}_{3} \\
(\%)\end{array}$ \\
\hline $23-5,109$ & 526.09 & 11.1 & 0.0 & 92 \\
\hline $57-1,48$ & 823.48 & 10.5 & 0.0 & 87 \\
\hline $\begin{array}{l}58-1,120 \\
58-2,140 \\
58-3,131 \\
58-4,64\end{array}$ & $\begin{array}{l}828.20 \\
829.90 \\
831.31 \\
832.14\end{array}$ & $\begin{array}{l}2.9 \\
2.5 \\
2.3 \\
8.9\end{array}$ & $\begin{array}{l}0.0 \\
0.0 \\
0.1 \\
0.0\end{array}$ & $\begin{array}{l}24 \\
20 \\
19 \\
74\end{array}$ \\
\hline $\begin{array}{l}59-1,31 \\
59-2,42 \\
59-3,63\end{array}$ & $\begin{array}{l}832.31 \\
833.92 \\
835.63\end{array}$ & $\begin{array}{l}8.4 \\
2.5 \\
8.6\end{array}$ & $\begin{array}{l}0.0 \\
0.0 \\
0.0\end{array}$ & $\begin{array}{l}69 \\
20 \\
71\end{array}$ \\
\hline $\begin{array}{l}60-2,71 \\
60-2,98 \\
60-2,114 \\
60-2,128\end{array}$ & $\begin{array}{l}843.21 \\
843.48 \\
843.64 \\
843.78\end{array}$ & $\begin{array}{r}10.8 \\
0.2 \\
0.0 \\
0.0\end{array}$ & $\begin{array}{l}0.0 \\
0.1 \\
0.0 \\
0.0\end{array}$ & $\begin{array}{r}89 \\
0 \\
0 \\
0\end{array}$ \\
\hline $\begin{array}{l}62-2,36 \\
62-2,67 \\
62-2,127 \\
62-3,75 \\
62-3,119 \\
62-4,40\end{array}$ & $\begin{array}{l}861.86 \\
862.17 \\
862.77 \\
863.75 \\
864.19 \\
864.90\end{array}$ & $\begin{array}{r}9.5 \\
10.2 \\
9.2 \\
4.2 \\
9.1 \\
10.0\end{array}$ & $\begin{array}{l}0.0 \\
0.0 \\
0.0 \\
0.0 \\
0.0 \\
0.0\end{array}$ & $\begin{array}{l}79 \\
85 \\
76 \\
35 \\
76 \\
83\end{array}$ \\
\hline $\begin{array}{l}63-1,43 \\
63-3,46 \\
63-3,85 \\
63-4,102\end{array}$ & $\begin{array}{l}870.43 \\
873.46 \\
873.85 \\
875.52\end{array}$ & $\begin{array}{r}8.8 \\
9.0 \\
10.3 \\
11.0\end{array}$ & $\begin{array}{l}0.0 \\
0.0 \\
0.1 \\
0.0\end{array}$ & $\begin{array}{l}74 \\
74 \\
86 \\
92\end{array}$ \\
\hline $\begin{array}{l}64-1,136 \\
64-2,111 \\
64-5,16 \\
64-5,135\end{array}$ & $\begin{array}{l}880.36 \\
881.61 \\
885.16 \\
886.34\end{array}$ & $\begin{array}{r}10.4 \\
11.3 \\
7.9 \\
9.6\end{array}$ & $\begin{array}{l}0.0 \\
0.0 \\
0.0 \\
0.1\end{array}$ & $\begin{array}{l}87 \\
94 \\
66 \\
79\end{array}$ \\
\hline $\begin{array}{l}65-1,49 \\
65-2,118 \\
65-3,93\end{array}$ & $\begin{array}{l}888.49 \\
890.68 \\
891.93\end{array}$ & $\begin{array}{r}10.7 \\
9.9 \\
7.5\end{array}$ & $\begin{array}{l}0.0 \\
0.0 \\
0.1\end{array}$ & $\begin{array}{l}89 \\
82 \\
62\end{array}$ \\
\hline $\begin{array}{l}67-1,122 \\
67-3,101\end{array}$ & $\begin{array}{l}908.22 \\
911.01\end{array}$ & $\begin{array}{l}9.3 \\
7.6\end{array}$ & $\begin{array}{l}0.1 \\
0.0\end{array}$ & $\begin{array}{l}77 \\
63\end{array}$ \\
\hline $\begin{array}{l}68-2,19 \\
68-2,69 \\
68-2,140 \\
68-2,142 \\
68-2,144 \\
68-3,14 \\
68-3,16 \\
68-5,60\end{array}$ & $\begin{array}{l}917.69 \\
918.19 \\
918.90 \\
918.92 \\
918.94 \\
919.14 \\
919.16 \\
922.60\end{array}$ & $\begin{array}{r}7.7 \\
7.0 \\
8.8 \\
7.5 \\
10.1 \\
2.9 \\
4.6 \\
3.9\end{array}$ & $\begin{array}{l}0.0 \\
0.0 \\
0.0 \\
0.0 \\
0.0 \\
0.0 \\
0.0 \\
0.0\end{array}$ & $\begin{array}{l}64 \\
58 \\
73 \\
62 \\
83 \\
24 \\
38 \\
32\end{array}$ \\
\hline $\begin{array}{l}69-3,34 \\
69-4,101 \\
69-4,147\end{array}$ & $\begin{array}{l}928.34 \\
930.51 \\
930.97\end{array}$ & $\begin{array}{l}5.2 \\
8.6 \\
7.8\end{array}$ & $\begin{array}{l}0.0 \\
0.1 \\
0.1\end{array}$ & $\begin{array}{l}43 \\
72 \\
65\end{array}$ \\
\hline $\begin{array}{l}70-1,79 \\
70-1,104 \\
70-1,122 \\
70-2,40 \\
70-2,53 \\
70-2,102 \\
70-4,1 \\
70-4,3\end{array}$ & $\begin{array}{l}935.79 \\
936.04 \\
936.22 \\
936.90 \\
937.03 \\
937.52 \\
939.51 \\
939.53\end{array}$ & $\begin{array}{r}7.9 \\
10.6 \\
4.0 \\
3.7 \\
11.1 \\
7.5 \\
10.9 \\
11.4\end{array}$ & $\begin{array}{l}0.0 \\
0.0 \\
0.1 \\
0.0 \\
0.0 \\
0.1 \\
0.0 \\
0.0\end{array}$ & $\begin{array}{l}66 \\
88 \\
33 \\
31 \\
92 \\
62 \\
90 \\
94\end{array}$ \\
\hline $71-2,98$ & 946.48 & 11.4 & 0.0 & 95 \\
\hline $72-2,0$ & 954.50 & 4.8 & 0.0 & 40 \\
\hline $\begin{array}{l}73-1,145 \\
73-2,30 \\
73-2,33 \\
73-2,36 \\
73-2,81\end{array}$ & $\begin{array}{l}963.45 \\
963.80 \\
963.83 \\
963.86 \\
964.31\end{array}$ & $\begin{array}{r}11.3 \\
10.7 \\
7.3 \\
5.7 \\
11.4\end{array}$ & $\begin{array}{l}0.0 \\
0.0 \\
0.0 \\
0.0 \\
0.0\end{array}$ & $\begin{array}{l}94 \\
89 \\
61 \\
47 \\
94\end{array}$ \\
\hline $74-1,137$ & 972.37 & 10.8 & 0.0 & 89 \\
\hline
\end{tabular}


TABLE 1 -Continued

\begin{tabular}{ccccc}
\hline $\begin{array}{c}\text { Core, Section, } \\
\text { Top of } \\
\text { Interval } \\
(\mathrm{cm})\end{array}$ & $\begin{array}{c}\text { Hole } \\
\text { Depth } \\
(\mathrm{m})\end{array}$ & $\begin{array}{c}\text { Total } \\
\text { Carbon } \\
(\%)\end{array}$ & $\begin{array}{c}\text { Organic } \\
\text { Carbon } \\
(\%)\end{array}$ & $\begin{array}{c}\mathrm{CaCO}_{3} \\
(\%)\end{array}$ \\
\hline $75-1,106$ & 982.06 & 11.2 & 0.0 & 93 \\
$76-1,67$ & 990.67 & 11.0 & 0.0 & 92 \\
$76-2,100$ & 992.50 & 10.9 & 0.0 & 90 \\
$77-1,115$ & 1000.15 & 10.8 & 0.0 & 90 \\
$88-1,115$ & 1109.15 & 9.8 & 0.0 & 81 \\
$92-1,148$ & 1149.48 & 11.4 & 0.0 & 95 \\
$93-1,80$ & 1157.80 & 11.2 & 0.0 & 93 \\
$93-2,70$ & 1159.20 & 10.9 & 0.1 & 90 \\
$94-2,29$ & 1167.79 & 6.7 & 0.1 & 55 \\
$94-2,33$ & 1167.83 & 8.1 & 0.1 & 66
\end{tabular}

Site 169

$\begin{array}{llllr}7-1,41 & 192.41 & 0.0 & 0.0 & 0 \\ 7-1,147 & 193.47 & 4.9 & 0.0 & 41\end{array}$

Site 170

$\begin{array}{lrrrr}2-2,50 & 3.00 & 0.0 & 0.0 & 0 \\ 2-4,50 & 6.00 & 0.0 & 0.0 & 0 \\ 6-4,97 & 106.47 & 0.0 & 0.0 & 0 \\ 6-4,126 & 106.76 & 7.9 & 0.0 & 65 \\ 6-4,140 & 106.90 & 10.0 & 0.0 & 83 \\ 6-5,100 & 108.00 & 1.5 & 0.0 & 12 \\ 6-6,86 & 109.36 & 8.8 & 0.0 & 73 \\ 6-6,117 & 109.67 & 5.4 & 0.0 & 45 \\ 6-6,119 & 109.69 & 0.2 & 0.0 & 1 \\ 7-2,50 & 112.00 & 9.2 & 0.0 & 77 \\ 7-3,10 & 113.10 & 8.3 & 0.0 & 69 \\ 7-3,30 & 113.30 & 0.0 & 0.0 & 0 \\ 7-3,107 & 114.07 & 8.5 & 0.0 & 71 \\ 8-1,25 & 120.25 & 8.1 & 0.0 & 68 \\ 8-1,59 & 120.59 & 0.1 & 0.0 & 1 \\ 15-1,123 & 185.23 & 0.9 & 0.0 & 7 \\ \mathbf{1 5 - 2 , 1 1 0} & 186.60 & 0.0 & 0.0 & 0 \\ 15-2,138 & 186.88 & 8.6 & 0.0 & 71\end{array}$

Site 171

\begin{tabular}{lrlll}
$1-2,20$ & 1.70 & 10.9 & 0.0 & 91 \\
$1-4,30$ & 4.80 & 11.2 & 0.0 & 93 \\
$1-6,20$ & 7.70 & 11.0 & 0.0 & 91 \\
$2-1,40$ & 22.40 & 10.5 & 0.0 & 88 \\
$2-2,20$ & 23.70 & 10.8 & 0.0 & 90 \\
$2-2,90$ & 24.40 & 11.0 & 0.0 & 92 \\
$2-3,107$ & 26.07 & 10.6 & 0.0 & 88 \\
$2-3,116$ & 26.16 & 10.5 & 0.0 & 88 \\
$2-4,50$ & 27.00 & 10.8 & 0.0 & 89 \\
$2-5,50$ & 28.50 & 11.0 & 0.0 & 91 \\
$3-1,30$ & 41.30 & 11.5 & 0.0 & 95 \\
$3-2,20$ & 42.70 & 11.4 & 0.0 & 95 \\
$3-3,50$ & 44.50 & 11.4 & 0.0 & 94 \\
$3-4,20$ & 45.70 & 10.5 & 0.0 & 87 \\
$3-6,20$ & 48.70 & 11.3 & 0.0 & 94 \\
\hline
\end{tabular}

TABLE 1 - Continued

\begin{tabular}{|c|c|c|c|c|}
\hline $\begin{array}{c}\text { Core, Section, } \\
\text { Top of } \\
\text { Interval } \\
\text { (cm) }\end{array}$ & $\begin{array}{l}\text { Hole } \\
\text { Depth } \\
\text { (m) }\end{array}$ & $\begin{array}{c}\text { Total } \\
\text { Carbon } \\
(\%)\end{array}$ & $\begin{array}{c}\text { Organic } \\
\text { Carbon } \\
(\%)\end{array}$ & $\begin{array}{c}\mathrm{CaCO}_{3} \\
(\%)\end{array}$ \\
\hline $4-1,30$ & 59.30 & 11.6 & 0.0 & 96 \\
\hline $4-2,50$ & 61.00 & 11.6 & 0.0 & 97 \\
\hline $4-3,50$ & 62.50 & 11.6 & 0.0 & 96 \\
\hline $4-4,50$ & 64.00 & 11.7 & 0.0 & 97 \\
\hline $4-5,50$ & 65.50 & 11.4 & 0.0 & 95 \\
\hline $4-6,50$ & 67.00 & 11.5 & 0.0 & 96 \\
\hline $5-1,20$ & 78.20 & 11.2 & 0.0 & 93 \\
\hline $5-3,20$ & 81.20 & 11.6 & 0.0 & 96 \\
\hline $5-4,40$ & 82.90 & 11.5 & 0.0 & 96 \\
\hline $5-5,20$ & 84.20 & 11.6 & 0.0 & 96 \\
\hline $6-2,50$ & 99.00 & 11.4 & 0.0 & 95 \\
\hline $8-3,30$ & 127.30 & 11.5 & 0.0 & 95 \\
\hline $8-5,20$ & 130.20 & 11.4 & 0.0 & 95 \\
\hline $9-3,50$ & 146.50 & 8.1 & 0.1 & 67 \\
\hline $9-5,69$ & 149.69 & 10.9 & 0.0 & 90 \\
\hline $10-4,30$ & 156.80 & 10.1 & 0.1 & 84 \\
\hline $11-4,20$ & 165.70 & 10.8 & 0.1 & 89 \\
\hline $13-4,20$ & 194.70 & 10.2 & 0.0 & 85 \\
\hline $15-2,120$ & 219.70 & 11.2 & 0.0 & 93 \\
\hline $15-4,20$ & 221.70 & 8.0 & 0.0 & 67 \\
\hline $15-6,120$ & 225.70 & 11.5 & 0.0 & 96 \\
\hline $16-1,33$ & 226.33 & 11.2 & 0.0 & 93 \\
\hline 164,20 & 230.70 & 4.6 & 0.0 & 38 \\
\hline $16-6,20$ & 233.70 & 10.7 & 0.0 & 89 \\
\hline $17-3,20$ & 239.20 & 11.3 & 0.0 & 94 \\
\hline $17-5,28$ & 242.28 & 11.2 & 0.0 & 93 \\
\hline $19-1,60$ & 255.60 & 11.0 & 0.0 & 91 \\
\hline $19-3,53$ & 258.53 & 11.2 & 0.0 & 93 \\
\hline $19-5,50$ & 261.50 & 10.8 & 0.0 & 90 \\
\hline $21-2,32$ & 274.82 & 9.0 & 0.0 & 75 \\
\hline $22-1,27$ & 282.27 & 5.6 & 0.1 & 47 \\
\hline $22-2,120$ & 284.70 & 6.8 & 0.0 & 56 \\
\hline $22-3,29$ & 285.29 & 5.1 & 0.1 & 42 \\
\hline $23-4,130$ & 297.80 & 5.8 & 0.1 & 47 \\
\hline $23-5,39$ & 298.39 & 4.8 & 0.2 & 39 \\
\hline $24-1,52$ & 301.52 & 2.9 & 0.3 & 22 \\
\hline $25-2,5$ & 311.55 & 11.2 & 2.8 & 70 \\
\hline $25-4,89$ & 315.39 & 8.5 & 0.2 & 69 \\
\hline $25-5,12$ & 316.12 & 6.7 & 0.3 & 53 \\
\hline $26-1,36$ & 320.36 & 2.5 & 0.1 & 20 \\
\hline $26-2,107$ & 322.57 & 6.4 & 0.2 & 52 \\
\hline $26-2,147$ & 322.97 & 6.8 & 0.3 & 54 \\
\hline $26-3,43$ & 323.43 & 1.4 & 0.2 & 10 \\
\hline $26-3,61$ & 323.61 & 1.0 & 0.1 & 8 \\
\hline $26-3,119$ & 324.19 & 9.5 & 2.3 & 60 \\
\hline $26-3,139$ & 324.39 & 3.4 & 0.2 & 27 \\
\hline $28-2,20$ & 339.70 & 4.2 & 0.0 & 35 \\
\hline $28-2,73$ & 340.23 & 3.0 & 0.0 & 24 \\
\hline $28-2,141$ & 340.91 & 6.6 & 0.0 & 55 \\
\hline $29-2,60$ & 349.10 & 11.9 & 0.0 & 99 \\
\hline
\end{tabular}

